Article

\title{
Energy-Efficient Clusters for Object Tracking Networks
}

\author{
Yang-Hsin Fan \\ Department of Computer Science and Information Engineering, National Taitung University, \\ Taitung 95092, Taiwan; yhfan@nttu.edu.tw; Tel.: +886-89-517605
}

Received: 31 May 2018; Accepted: 31 July 2018; Published: 2 August 2018

\begin{abstract}
Smart cities have hundreds of thousands of devices for tracking data on crime, the environment, and traffic (such as data collected at crossroads and on streets). This results in higher energy usage, as they are recording information persistently and simultaneously. Moreover, a single object tracking device, on a corner at an intersection for example has a limited scope of view, so more object tracking devices are added to broaden the view. As an increasing number of object tracking devices are constructed on streets, their efficient energy consumption becomes a significant issue. This work is concerned with decreasing the energy required to power these systems, and proposes energy-efficient clusters (EECs) of object tracking systems to achieve energy savings. First, we analyze a current object tracking system to establish an equivalent model. Second, we arrange the object tracking system in a cluster structure, which facilitates the evaluation of energy costs. Third, the energy consumption is assessed as either dynamic or static, which is a more accurate system for determining energy consumption. Fourth, we analyze all possible scenarios of the object's location and the resulting energy consumption, and derive a number of formulas for the fast computation of energy consumption. Finally, the simulation results are reported. These results show the proposed EEC is an effective way to save energy, compared with the energy consumption benchmarks of current technology.
\end{abstract}

Keywords: object tracking networks; energy saving; embedded system

\section{Introduction}

In Taiwan, cameras are very common at crossroads, streets, corners, and underpasses for the purposes of monitoring potential criminal activity, gathering data on the environment, and collecting information regarding traffic. Figure 1 shows an example of an intersection with a set of cameras in Taitung City. Four cameras are deployed at the eastern intersection, which is marked in red, while another four are set up in the west. These devices gradually may be upgraded to object tracking systems, in order to track car accidents, potentially record information from terrorist attacks, and help city security. For the foreseeable future, an increasing number of object tracking devices will continually be implemented and deployed to surveil traffic, and work towards the prevention of damage within the city.

In recent years, advanced integrated circuit technology has led to the manufacturing of powerful multicores and multiprocessors for consumer electronics. Performance and response time of the technology involved has improved, in comparison to previous technologies, such as a single controller or processor. Moreover, concurrent software runs on parallel architecture, which leads to high-throughput for diverse services. Multiprocessors, with integrated power, and concurrently run software have allowed the technology within traditional cameras to evolve, allowing for the invention of auto-follow cameras and object tracking systems. For example, in the commercial field, the company Movensee [1] has marketed auto-follow cameras to sports, rehearsals, and vloggers. Another company, Rayoptic [2], develops smart multiple-target tracking cameras for intrusion alarms, line-crossing alarms, and tracking suspicious activity. In the academic field, on the other hand, researchers have applied object tracking technology to develop intelligent surveillance systems [3] for elderly care, and smart home systems [4] for baby care. 


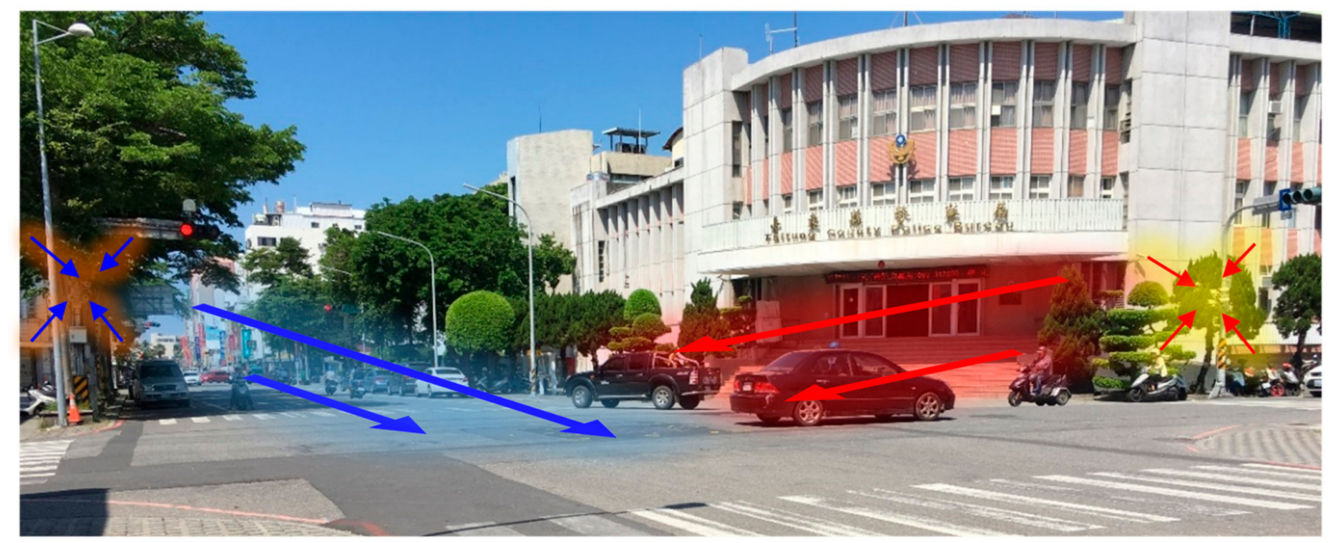

Figure 1. Cameras at an intersection.

Once an object tracking device has been set up, it can record information continuously. Therefore, it is anticipated that a great amount of energy will be consumed due to the persistent recording of data. Moreover, the number of object tracking devices is increasing substantially, due to the growing attention to issues related to security and environmental data collection. As new object tracking devices are implemented, even more energy will be consumed.

\section{Object Tracking Networks}

Current surveillance technology uses a large number of cameras, in order to increase the monitoring system's range for tracking traffic information or city security. Even on a small street corner, there are generally four or more cameras in place to avoid losing target objects. Each camera is responsible for a specific angle, and is functioning continuously.

Active cameras with wide angles take better panorama photos than general traditional cameras. This has increased the ability to capture images at angles. To display and resize images, these cameras often have powerful digital signal image processors. However, a camera cannot meet the demand of object tracking applications with only a wide angle and image processing, as it lacks technologies such as: Object tracking motion detection algorithms [5-7], the Markov Chain research model [8-12], optical flow technology [13-15], background model approach [16,17], and kernel-based method [18-21]. These systems also use a large amount of energy. To solve this problem Pooranian et al. [22] proposed an optimization model to schedule controlled and uncontrolled tasks, distributed energy resources (DER), as well as mixed integer linear programming (MILP). In fog computing research, Baccarelli et al. [23] presented a novel paradigm named "fog of everything" (FoE) for energy-efficient network computing architectures and research. Simulation results showed that the delay and energy were improved by FoE. Naranjo et al. [24] presented a fog computing architecture network (FOCAN) to enhance services with low and efficient energy usage in devices. Once these solutions are developed, integration of wide-range, high-resolution technologies into active cameras will allow the object tracking systems to work more intelligently.

In addition to intelligence, a desirable feature for object tracking system is efficient energy consumption. In a current surveillance system, a set of cameras are recording data continuously and simultaneously. Energy saving is hard to achieve, because too many cameras work simultaneously. Moreover, it is obvious that a small street corner equipped with four cameras or more creates a huge amount of data. Data recorded by each camera are highly similar, if not duplications of one another, which not only raises the cost for extracting images, but also significantly decreased energy utilization. However, consuming this much energy is not necessary if required images can be captured by only one camera. Therefore, a suitable camera arrangement would allow events to be captured by fewer cameras. The amount of energy consumption is gradually decreasing as a result of a reduction in the number of camera working simultaneously. Using Figure 1 as an example, the cameras on the right (in red) should 
be arranged appropriately in order to alternately record data, rather than simultaneously record. At the same time, cameras on the left (in blue) could sequentially capture images, instead of continuously recording data. Similarly, the other cameras in this figure could work in the same way.

A surveillance system set at a street corner to form a clustered architecture in Taitung City is shown in Figure 1. Similar streets with such surveillance systems (four clusters located on each corner) are ubiquitous in Taitung City, even though the citizens of the city are dedicated to improving energy consumption. In fact, a similar surveillance system is also deployed in many cities. Based on the scenario in Figure 1, we defined an equivalent model, shown in Figure 2 and named object tracking networks (OTNs), consisting of A, B, C, and D clusters located on the corner of a crossroad. Each cluster A-D consists of a number of object tracking devices, all of which consume energy continuously to tracking objects. From the perspective of energy utilization, this is absolutely inefficient as each device consumes energy simultaneously. Consequently, we propose an energy-efficient cluster for object tracking networks to achieve energy savings. In this study, we focused on the issue of energy consumption for an OTN cluster, making the following assumptions: (i) The object tracking device has an active mode (or working mode), and a standby mode, which consume dynamic and static energy, respectively; (ii) dynamic and static energy consumption of object tracking devices can be measured; (iii) each device has a wide angle for tracking objects; (iv) any object tracking device can be delegated to conduct surveillance during specific periods; and (v) energy consumption is ignored among devices during the swap between modes.

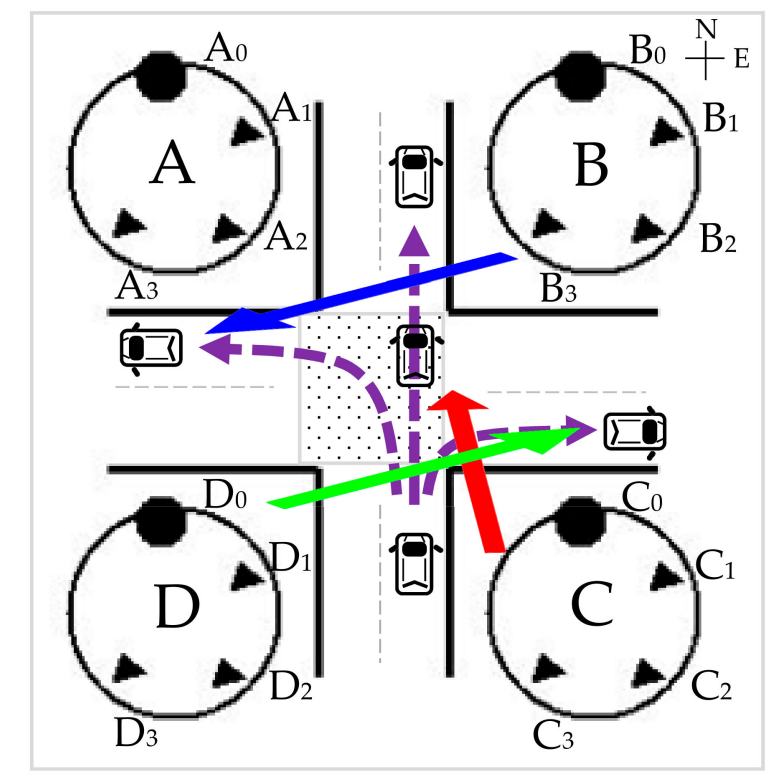

Figure 2. Equivalent model of object tracking networks (OTNs) with the target object approaching from the south.

In an intersection, the target object may be located in the east, west, south, and north. Different locations of target objects will activate the appropriate object tracking device in the cluster. For example, if the target object approaches from the south it may go straight, turn right, or turn left. No matter where the object goes, it can be tracked by the OTN, which has been set at the corner. For instance, if the object goes straight, it is monitored via the object tracking devices in cluster $\mathrm{C}$, and the other object tracking devices in clusters A, B, and D are set to standby mode as they are not needed for surveillance. If the object turns right, it activates the object tracking devices in cluster $\mathrm{D}$, and the object tracking devices in clusters $\mathrm{A}, \mathrm{B}$, and $\mathrm{C}$ are set to standby mode. If the object turns left, it activates the object tracking devices in cluster $\mathrm{B}$, and the other object tracking devices in clusters $\mathrm{A}$, $\mathrm{C}$, and D are set to standby mode. 
Generally, standby mode consumes less energy than when the device is active because the former consumes static energy, and the latter uses dynamic energy. Such research was discussed in References [25-27]. In the aforementioned scenarios, the object tracking devices in cluster A are always in standby mode. Therefore, its energy consumption will be less than clusters B, C, and D. Conversely, the other three clusters, which are active, respectively, depending on the direction of the target object, consume dynamic energy. We define $E_{\mathrm{d}}$ and $E_{\mathrm{s}}$ as the dynamic and static energy use as follows:

$$
\begin{gathered}
E_{\mathrm{d}}=P_{\mathrm{d}} \times T_{\mathrm{d}}, \\
E_{\mathrm{s}}=P_{\mathrm{s}} \times T_{\mathrm{s}},
\end{gathered}
$$

where $P_{\mathrm{d}}$ and $P_{\mathrm{s}}$ are dynamic and static power, and $T_{\mathrm{d}}$ and $T_{\mathrm{s}}$ are dynamic and static execution time.

First, we analyze one object approaching from the south in Figure 2. There are three possible paths: Turning left and then proceeding west, in which case dynamic energy is consumed in cluster $B$ while the object completes the left turn while clusters A, C, and D consume static energy; proceeding straight, causing dynamic energy consumption in cluster $C$, but static energy consumption in clusters $\mathrm{A}, \mathrm{B}$, and D; or the object could turn right, leading to dynamic energy consumption in cluster D, but static energy is used in cluster A-C. Table 1 summaries the energy consumption patterns for the various clusters for the three paths.

Table 1. Energy consumption of object tracking clusters for an object approaching from the south.

\begin{tabular}{cccc}
\hline \multirow{2}{*}{ Cluster } & \multicolumn{3}{c}{ Energy Consumption } \\
\cline { 2 - 4 } & Turn Left & Go Straight & Turn Right \\
\hline A & Static & Static & Static \\
B & Dynamic & Static & Static \\
C & Static & Dynamic & Static \\
D & Static & Static & Dynamic \\
\hline
\end{tabular}

Clusters consuming dynamic and static energy in Figure 2 are summarized in Table 1. It is helpful to further evaluate energy consumption of object tracking devices. According to Section 2, a cluster consists of a set of object tracking devices. The number of object tracking devices is either fixed or variant. Four object tracking devices for each cluster are named $X_{0}, X_{1}, X_{2}$, and $X_{3}$, in accordance with Figure 1. Then, the energy consumption of each object tracking device is evaluated for the target object going straight in Table 2 by applying Equations (1) and (2), and the data in Table 1. As a result, the object tracking devices, $C_{0}-C_{3}$, in cluster $C$ are active, which consumes dynamic energy rather than static energy. Devices in clusters A, B, and D are triggered to enter standby mode, where they consume static energy as opposed to dynamic energy.

According to Table 2, the dynamic energy consumption of cluster $C$ is defined as follows:

$$
\sum_{i} E_{\mathrm{d}_{i}}
$$

where $i$ is the number of object tracking devices. On the other hand, the static energy consumption of clusters $\mathrm{A}, \mathrm{B}$, and $\mathrm{D}$ is defined in the following:

$$
\sum_{k} \sum_{j \neq i} E_{\mathrm{s}_{j}}
$$

where $j$ is the number of object tracking devices and $j \neq i$, and $k$ is the number of clusters. The sum of energy consumption of the OTN is defined as follows:

$$
\sum_{i} E_{\mathrm{d}_{i}}+\sum_{k} \sum_{j \neq i} E_{\mathrm{s}_{j}}
$$


Table 2. Cluster $C$ energy consumption for a target object that goes straight.

\begin{tabular}{cccc}
\hline \multirow{3}{*}{ Cluster } & \multirow{2}{*}{ Object Tracking Devices } & \multicolumn{2}{c}{ Energy Consumption } \\
\cline { 2 - 4 } & & Dynamic & \multicolumn{1}{c}{ Static } \\
\hline \multirow{2}{*}{$\mathrm{A}$} & $\mathrm{A}_{0}$ & 0 & $P_{\mathrm{s}, \mathrm{A}_{0}} \times T_{\mathrm{s}, \mathrm{A}_{0}}$ \\
& $\mathrm{~A}_{1}$ & 0 & $P_{\mathrm{s}, \mathrm{A}_{1}} \times T_{\mathrm{s}, \mathrm{A}_{1}}$ \\
& $\mathrm{~A}_{2}$ & 0 & $P_{\mathrm{s}, \mathrm{A}_{2}} \times T_{\mathrm{s}, \mathrm{A}_{2}}$ \\
& $\mathrm{~A}_{3}$ & 0 & $P_{\mathrm{s}, \mathrm{A}_{3}} \times T_{\mathrm{s}, \mathrm{A}_{3}}$ \\
\hline $\mathrm{B}$ & $\mathrm{B}_{0}$ & 0 & $P_{\mathrm{s}, \mathrm{B}_{0}} \times T_{\mathrm{s}, \mathrm{B}_{0}}$ \\
& $\mathrm{~B}_{1}$ & 0 & $P_{\mathrm{s}, \mathrm{B}_{1}} \times T_{\mathrm{s}, \mathrm{B}_{1}}$ \\
& $\mathrm{~B}_{2}$ & 0 & $P_{\mathrm{s}, \mathrm{B}_{2}} \times T_{\mathrm{s}, \mathrm{B}_{2}}$ \\
& $\mathrm{~B}_{3}$ & 0 & $P_{\mathrm{s}, \mathrm{B}_{3}} \times T_{\mathrm{s}, \mathrm{B}_{3}}$ \\
\hline $\mathrm{C}$ & $\mathrm{C}_{0}$ & $P_{\mathrm{d}, \mathrm{C}_{0}} \times T_{\mathrm{d}, \mathrm{C}_{0}}$ & \\
& $\mathrm{C}_{1}$ & $P_{\mathrm{d}, \mathrm{C}_{1}} \times T_{\mathrm{d}, \mathrm{C}_{1}}$ & 0 \\
& $\mathrm{C}_{2}$ & $P_{\mathrm{d}, \mathrm{C}_{2}} \times T_{\mathrm{d}, \mathrm{C}_{2}}$ & 0 \\
& $\mathrm{C}_{3}$ & $P_{\mathrm{d}, \mathrm{C}_{3}} \times T_{\mathrm{d}, \mathrm{C}_{3}}$ & 0 \\
\hline $\mathrm{D}$ & $\mathrm{D}_{0}$ & 0 & $P_{\mathrm{s}, \mathrm{D}_{0}} \times T_{\mathrm{s}, \mathrm{D}_{0}}$ \\
& $\mathrm{D}_{1}$ & 0 & $P_{\mathrm{s}, \mathrm{D}_{1}} \times T_{\mathrm{s}, \mathrm{D}_{1}}$ \\
& $\mathrm{D}_{2}$ & 0 & $P_{\mathrm{s}, \mathrm{D}_{2}} \times T_{\mathrm{s}, \mathrm{D}_{2}}$ \\
& $\mathrm{D}_{3}$ & 0 & $P_{\mathrm{s}, \mathrm{D}_{3}} \times T_{\mathrm{s}, \mathrm{D}_{3}}$ \\
\hline
\end{tabular}

A second case is demonstrated in Figure 3, in which the target object approaches from the west. If it goes straight, cluster D will consume dynamic energy and the other clusters will consume static energy. Another path is to proceed south, meaning cluster A consumes dynamic and the other clusters use static energy. If the objects turns left, cluster $C$ will consume dynamic energy, and the other clusters to consume static energy. These clusters and their patterns of energy consumption are listed in Table 3. A third case is the object approaching from the north, as exhibited in Figure 4. The resulting energy consumption is reported in Table 4. Finally, the target object starting from the east is depicted in Figure 5 . The energy consumption resulting from this case is presented in Table 5.

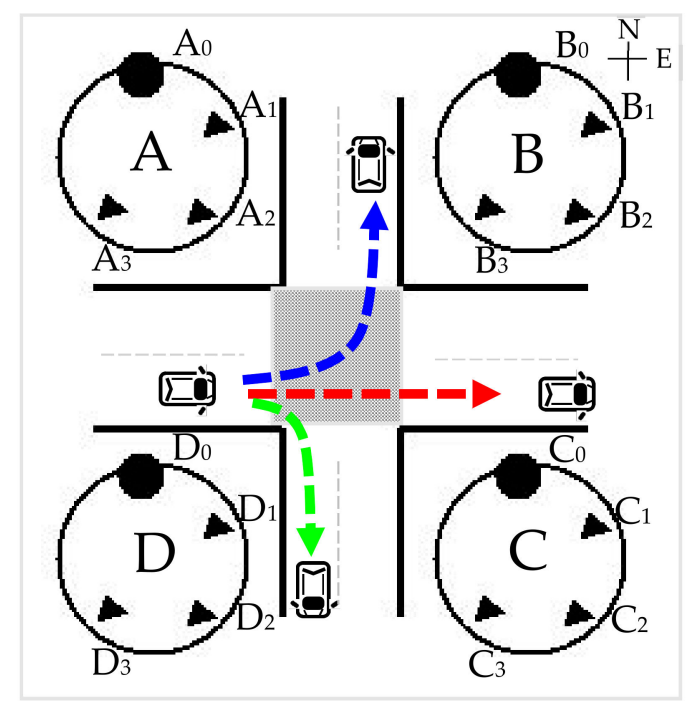

Figure 3. Equivalent model of object tracking networks with the object approaching from the west. 
Table 3. Energy consumption of object tracking clusters with the object approaching from the west.

\begin{tabular}{cccc}
\hline \multirow{2}{*}{ Cluster } & \multicolumn{3}{c}{ Energy Consumption } \\
\cline { 2 - 4 } & Turn Left & Go Straight & Turn Right \\
\hline A & Static & Static & Dynamic \\
B & Static & Static & Static \\
C & Dynamic & Static & Static \\
D & Static & Dynamic & Static \\
\hline
\end{tabular}

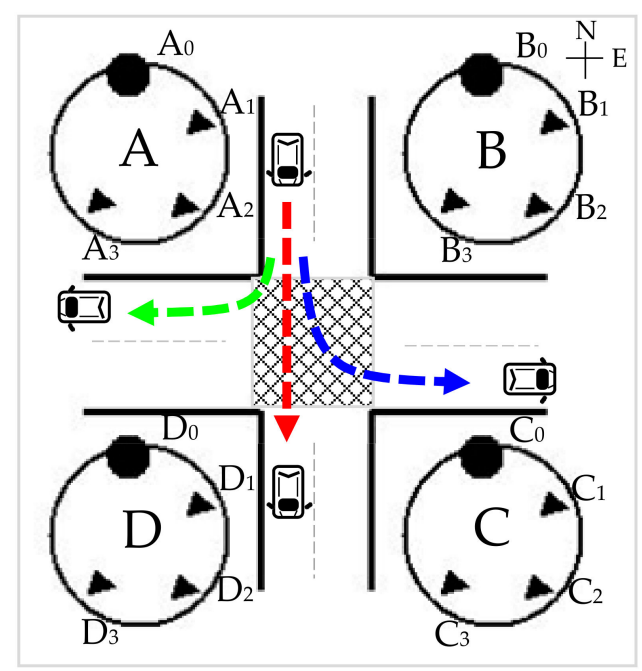

Figure 4. Equivalent model of object tracking networks with the object approaching from the north.

Table 4. Energy consumption of object tracking clusters with the object approaching from the north.

\begin{tabular}{cccc}
\hline \multirow{2}{*}{ Cluster } & \multicolumn{3}{c}{ Energy Consumption } \\
\cline { 2 - 4 } & Turn Left & Go Straight & Turn Right \\
\hline A & Static & Dynamic & Static \\
B & Static & Static & Dynamic \\
C & Static & Static & Static \\
D & Dynamic & Static & Static \\
\hline
\end{tabular}

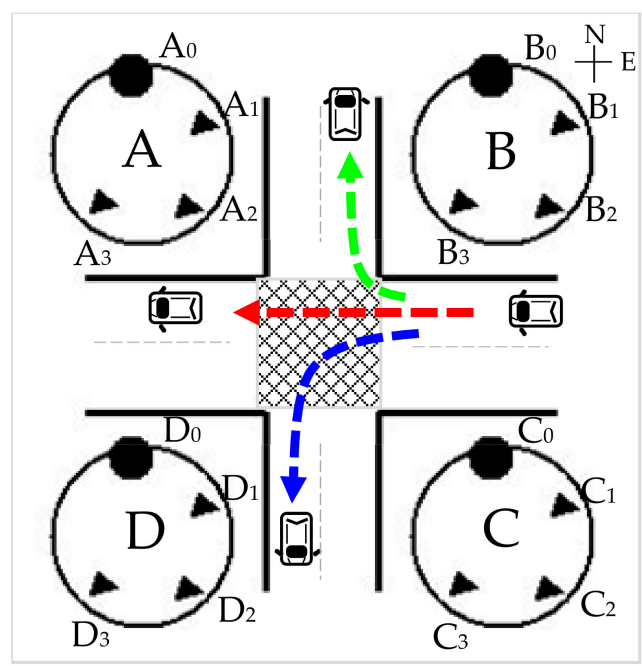

Figure 5. Equivalent model of object tracking networks with the object approaching from the east. 
Table 5. Energy consumption of object tracking clusters with the object approaching from the east.

\begin{tabular}{cccc}
\hline \multirow{2}{*}{ Cluster } & \multicolumn{3}{c}{ Energy Consumption } \\
\cline { 2 - 4 } & Turn Left & Go Straight & Turn Right \\
\hline A & Dynamic & Static & Static \\
B & Static & Dynamic & Static \\
C & Static & Static & Dynamic \\
D & Static & Static & Static \\
\hline
\end{tabular}

After analyzing all possible cases, the energy consumption of the object tracking network is defined in the following:

$$
\sum_{n}\left(\sum_{i} E_{\mathrm{d}_{i}}+\sum_{k} \sum_{j \neq i} E_{\mathrm{s}_{j}}\right)
$$

where $n$ is the number of OTNs.

\section{Simulation}

Figure 6 shows a scenario for the object tracking network. There are five object tracking networks, which are located in the northwest, northeast, middle, southwest, and southeast. Each OTN consists of four clusters, and each cluster has four object tracking devices. The number of object tracking devices is primarily based on the intersection at the Taitung county police bureau in Figure 1. The specifications of energy consumption for each object tracking device is according to the AHD 4MP surveillance cameras from Kingnet Technology Company (Taipei, Taiwan) [28]. The voltage is direct-current voltage $12 \mathrm{~V}$ and the current is between $100 \mathrm{~mA}$ and $350 \mathrm{~mA}$.

Table 6 displays the randomly generated $E_{\mathrm{d}}$ and $E_{\mathrm{S}}$ for OTN 1,3, and 4 . They are generated based on the following rules: (i) The value of the power generated is between 1.2 and $4.2 \mathrm{~W}$, in accordance with AHD 4MP surveillance camera specifications [28]; (ii) the dynamic energy consumption is higher than the static energy consumption; and (iii) the execution time is set as one unit to easily calculate the proportion of energy consumption.

The target being tracked approaches from the north and travels along the red dotted line in Figure 6. It sequentially passes by OTN 1, 3, then 4 . Therefore, clusters A, B, C, and D in OTN 1 will be activated when the tracked target arrives in their surveillance area. According to the direction of the target, cluster $B$ is activated first at time $0\left(t_{0}\right)$, and all cluster $B$ object tracking devices, $B_{0}-B_{3}$, consume dynamic energy. Clusters A, C, and D in OTN 1, and all clusters in OTN 2-5, are in standby mode-resulting in static energy consumption. Second, the target arrives in cluster A's area, where the set of object tracking devices are named $A_{0}, A_{1}, A_{2}$, and $A_{3}$. The four devices execute the object tracking job, resulting in dynamic energy consumption. Conversely, clusters $\mathrm{B}-\mathrm{T}$ consume static energy. Both dynamic and static energy consumption are recorded at time $1\left(\mathrm{t}_{1}\right)$. Third, the target object leaves the OTN 1 area and goes to the OTN 3 region. Time 2 is designated $t_{2}$. Dynamic energy consumption occurs in cluster $\mathrm{L}$. Consequently, the object tracking devices, $\mathrm{L}_{0}, \mathrm{~L}_{1}, \mathrm{~L}_{2}$, and $\mathrm{L}_{3}$ consume dynamic energy. In contrast, the rest of the clusters consume static energy. Fourth, the object turns right from cluster $\mathrm{L}$. The scope of surveillance is in cluster I, whose set of object tracking devices are designated $\mathrm{I}_{0}, \mathrm{I}_{1}, \mathrm{I}_{2}$, and $\mathrm{I}_{3}$, which are, enabled to track the target, resulting in their dynamic energy consumption at time 3 (i.e., $t_{3}$ ). All clusters, excluding cluster I, consume static energy. Finally, the target turns right and then arrives at the intersection of OTN 4 . Cluster $\mathrm{N}$ enters into service at time 4 , also named $\mathrm{t}_{4}$. Dynamic energy consumption occurs in object tracking devices $\mathrm{N}_{0}, \mathrm{~N}_{1}, \mathrm{~N}_{2}$, and $\mathrm{N}_{3}$, while the other clusters consume static energy. 


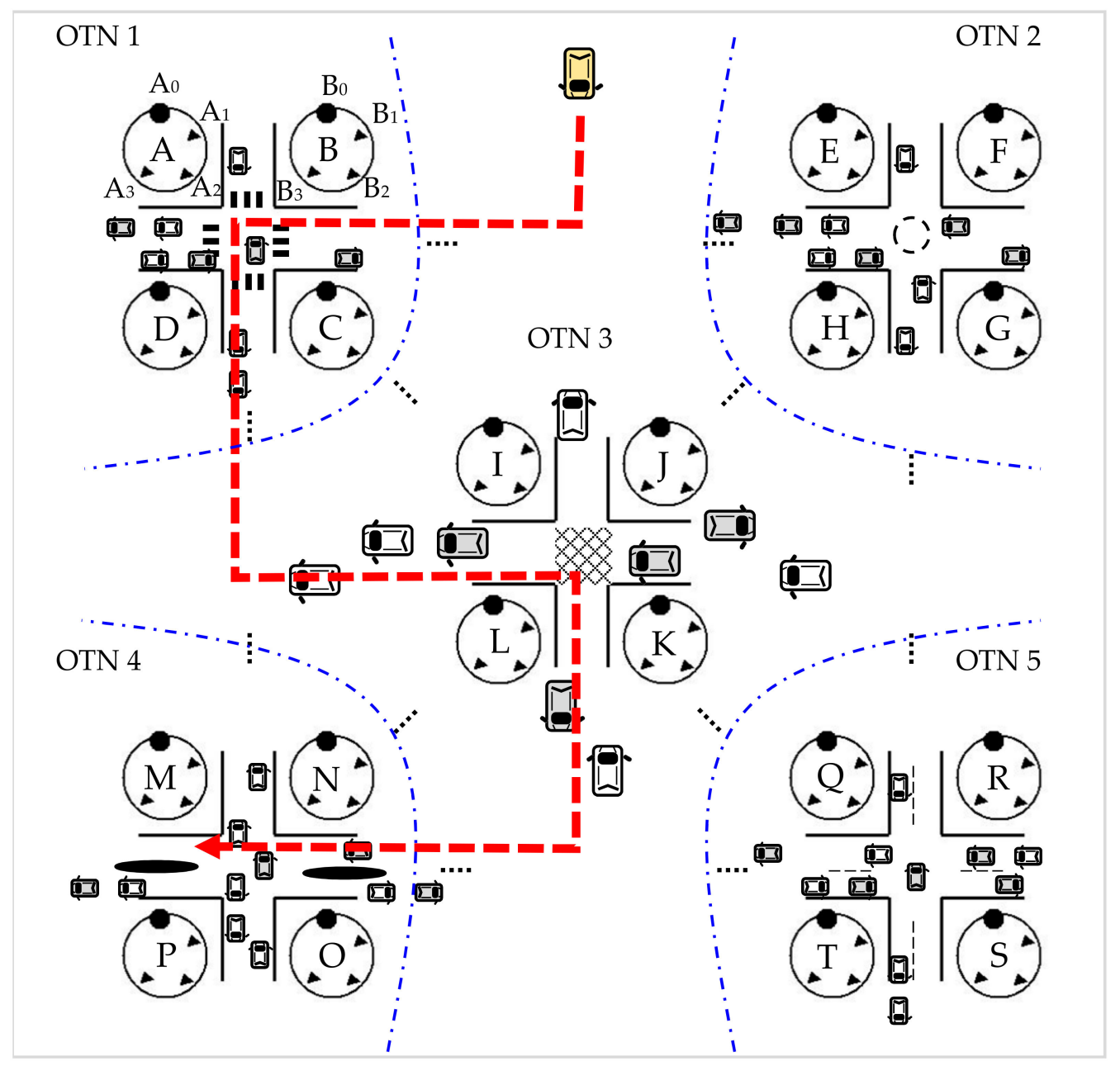

Figure 6. Clusters of energy consumption for object tracking networks.

Table 6. Parameters and values of energy consumption for OTN 1, 3, and 4.

\begin{tabular}{ccccccccc}
\hline \multicolumn{7}{c}{ Energy Consumption } \\
\hline Cluster & $\boldsymbol{E}_{\mathbf{d}}$ & $\boldsymbol{E}_{\mathbf{s}}$ & Cluster & $\boldsymbol{E}_{\mathbf{d}}$ & $\boldsymbol{E}_{\mathbf{s}}$ & Cluster & $\boldsymbol{E}_{\mathbf{d}}$ & $\boldsymbol{E}_{\mathbf{s}}$ \\
\hline $\mathrm{A}_{0}$ & 3.36 & 0.61 & $\mathrm{I}_{0}$ & 3.81 & 0.53 & $\mathrm{M}_{0}$ & 2.82 & 0.61 \\
$\mathrm{~A}_{1}$ & 3.36 & 0.50 & $\mathrm{I}_{1}$ & 3.97 & 0.77 & $\mathrm{M}_{1}$ & 2.88 & 0.37 \\
$\mathrm{~A}_{2}$ & 1.11 & 0.93 & $\mathrm{I}_{2}$ & 3.59 & 0.19 & $\mathrm{M}_{2}$ & 3.96 & 0.32 \\
$\mathrm{~A}_{3}$ & 3.64 & 0.63 & $\mathrm{I}_{3}$ & 3.90 & 0.41 & $\mathrm{M}_{3}$ & 1.34 & 0.94 \\
$\mathrm{~B}_{0}$ & 3.56 & 0.38 & $\mathrm{~J}_{0}$ & 1.81 & 0.28 & $\mathrm{~N}_{0}$ & 2.62 & 0.77 \\
$\mathrm{~B}_{1}$ & 2.73 & 0.67 & $\mathrm{~J}_{1}$ & 1.53 & 0.41 & $\mathrm{~N}_{1}$ & 3.24 & 0.49 \\
$\mathrm{~B}_{2}$ & 1.88 & 0.14 & $\mathrm{~J}_{2}$ & 2.37 & 0.26 & $\mathrm{~N}_{2}$ & 2.61 & 0.95 \\
$\mathrm{~B}_{3}$ & 3.90 & 0.88 & $\mathrm{~J}_{3}$ & 1.85 & 0.23 & $\mathrm{~N}_{3}$ & 3.66 & 0.05 \\
$\mathrm{C}_{0}$ & 1.40 & 0.48 & $\mathrm{~K}_{0}$ & 2.11 & 0.78 & $\mathrm{O}_{0}$ & 3.26 & 0.18 \\
$\mathrm{C}_{1}$ & 3.18 & 0.26 & $\mathrm{~K}_{1}$ & 2.48 & 0.79 & $\mathrm{O}_{1}$ & 2.69 & 0.02 \\
$\mathrm{C}_{2}$ & 3.18 & 0.40 & $\mathrm{~K}_{2}$ & 2.44 & 0.59 & $\mathrm{O}_{2}$ & 3.57 & 0.60 \\
$\mathrm{C}_{3}$ & 1.42 & 0.87 & $\mathrm{~K}_{3}$ & 2.78 & 0.31 & $\mathrm{O}_{3}$ & 2.62 & 0.65 \\
$\mathrm{D}_{0}$ & 4.00 & 0.79 & $\mathrm{~L}_{0}$ & 2.85 & 0.36 & $\mathrm{P}_{0}$ & 1.87 & 0.43 \\
$\mathrm{D}_{1}$ & 3.12 & 0.59 & $\mathrm{~L}_{1}$ & 3.29 & 0.80 & $\mathrm{P}_{1}$ & 2.97 & 0.65 \\
$\mathrm{D}_{2}$ & 2.52 & 0.35 & $\mathrm{~L}_{2}$ & 1.86 & 0.25 & $\mathrm{P}_{2}$ & 3.62 & 0.07 \\
$\mathrm{D}_{3}$ & 1.80 & 0.55 & $\mathrm{~L}_{3}$ & 2.63 & 0.41 & $\mathrm{P}_{3}$ & 3.53 & 0.11 \\
\hline
\end{tabular}


Table 7 shows the simulation results with four cameras for the scenario in Figure 6. We compared current technology and the proposed approach. Firstly, with current technology, clusters (A-T) would work continuously and simultaneously, resulting in the dynamic energy consumption shown in column 2 . Conversely, the proposed approach only consumes dynamic energy, $E_{\mathrm{d}}$, in cluster $\mathrm{B}$. Therefore, the $E_{\mathrm{d}}$ in cluster $B$ is the sum of $B_{0}, B_{1}, B_{2}$, and $B_{3}$, whose data are shown in Table 6 . The other clusters work on standby mode; therefore, their $E_{\mathrm{d}}$ value is 0 . The $E_{\mathrm{d}}$ values for all clusters of the proposed method are reported in column 3 . All clusters, excluding cluster $\mathrm{B}$, consume static energy $E_{\mathrm{s}}$. Therefore, the $E_{\mathrm{s}}$ of cluster $\mathrm{B}$ is 0 , and that of the other clusters is the sum of $E_{\mathrm{S}}$ for each respective object tracking device. The values of $E_{\mathrm{s}}$ for all clusters are recorded in column 4 . Secondly, the target object arrives in another surveillance area at $t_{1}$. To reflect current technology, where clusters are continuously and simultaneously monitored, the energy consumption is calculated by $E_{\mathrm{d}}$, shown in column 5 . On the other hand, the proposed approach recognizes the working cluster as cluster $A$. Consequently, object tracking devices $A_{0}, A_{1}, A_{2}$, and $A_{3}$ in cluster A consume dynamic energy and the other clusters consume static energy. Both $E_{\mathrm{d}}$ and $E_{\mathrm{s}}$ are presented in columns 6 and 7. Next, the target object passes sequentially through the areas for which clusters $\mathrm{L}, \mathrm{I}$, and $\mathrm{N}$ are responsible. Their dynamic and static energy consumption is shown in columns 9 , $10,12,13,15$, and 16, respectively. Figures 7 and 8 show the simulation results for energy consumption with three and two cameras, in comparison with current technology.

In order to present the effectiveness of the energy-efficient cluster (EEC) approach, we computed an improvement ratio, comparing the EEC approach to current technology. At $t_{0}$, the EEC approach consumes $50.09 \mathrm{~J}$ of total energy and the current technology consumes $218.36 \mathrm{~J}$. This is an improvement ratio of $77.06 \%$. At $t_{1}$, the energy consumption for the EEC approach and current technology is, respectively, $48.89 \mathrm{~J}$ and $218.36 \mathrm{~J}$. The improvement ratio is $77.61 \%$. The other surveillance areas for the target object achieve, respectively, improvement ratios of $77.61 \%, 75.52 \%$, and $77.12 \%$ at $\mathrm{T}_{2}-\mathrm{T}_{4}$.

Table 7. Comparison of energy consumption of four cameras between the current method and the energy-efficient cluster (EEC) approach.

\begin{tabular}{|c|c|c|c|c|c|c|c|c|c|c|c|c|c|c|c|}
\hline \multirow{3}{*}{$\begin{array}{c}\text { Time } \\
\text { Clusters }\end{array}$} & \multicolumn{15}{|c|}{ Energy Consumption } \\
\hline & \multicolumn{3}{|c|}{$t_{0}$} & \multicolumn{3}{|c|}{$t_{1}$} & \multicolumn{3}{|c|}{$t_{2}$} & \multicolumn{3}{|c|}{$t_{3}$} & \multicolumn{3}{|c|}{$\mathbf{t}_{4}$} \\
\hline & \multirow{2}{*}{$\frac{\text { Cur. }}{E_{\mathrm{d}}}$} & \multicolumn{2}{|c|}{ Our } & \multirow{3}{*}{$\begin{array}{l}\text { Cur. } \\
E_{\mathrm{d}}\end{array}$} & \multicolumn{2}{|c|}{ Our } & \multirow{3}{*}{$\begin{array}{c}\text { Cur. } \\
E_{\mathrm{d}} \\
11.47\end{array}$} & \multicolumn{2}{|c|}{ Our } & \multirow{3}{*}{$\begin{array}{c}\text { Cur. } \\
E_{\mathrm{d}}\end{array}$} & \multicolumn{2}{|c|}{ Our } & \multirow{3}{*}{$\begin{array}{c}\text { Cur. } \\
E_{\mathrm{d}} \\
11.47\end{array}$} & \multicolumn{2}{|c|}{ Our } \\
\hline & & $E_{\mathrm{d}}$ & $E_{\mathrm{S}}$ & & $E_{\mathrm{d}}$ & $E_{\mathrm{S}}$ & & $E_{\mathrm{d}}$ & $E_{\mathrm{S}}$ & & $E_{\mathrm{d}}$ & $E_{\mathrm{S}}$ & & $E_{\mathrm{d}}$ & $E_{\mathrm{S}}$ \\
\hline A & 11.47 & 0 & 2.67 & & 11.47 & 0 & & 0 & 2.67 & & 0 & 2.67 & & 0 & 2.67 \\
\hline $\mathrm{B}$ & 12.07 & 12.07 & 0 & 12.07 & 0 & 2.07 & 12.07 & 0 & 2.07 & 12.07 & 0 & 2.07 & 12.07 & 0 & 2.07 \\
\hline $\mathrm{C}$ & 9.18 & 0 & 2.01 & 9.18 & 0 & 2.01 & 9.18 & 0 & 2.01 & 9.18 & 0 & 2.01 & 9.18 & 0 & 2.01 \\
\hline $\mathrm{D}$ & 11.44 & 0 & 2.28 & 11.44 & 0 & 2.28 & 11.44 & 0 & 2.28 & 11.44 & 0 & 2.28 & 11.44 & 0 & 2.28 \\
\hline $\mathrm{E}$ & 11.28 & 0 & 2.07 & 11.28 & 0 & 2.07 & 11.28 & 0 & 2.07 & 11.28 & 0 & 2.07 & 11.28 & 0 & 2.07 \\
\hline $\mathrm{F}$ & 12.23 & 0 & 1.86 & 12.23 & 0 & 1.86 & 12.23 & 0 & 1.86 & 12.23 & 0 & 1.86 & 12.23 & 0 & 1.86 \\
\hline G & 11.65 & 0 & 2.28 & 11.65 & 0 & 2.28 & 11.65 & 0 & 2.28 & 11.65 & 0 & 2.28 & 11.65 & 0 & 2.28 \\
\hline $\mathrm{H}$ & 9.16 & 0 & 2.53 & 9.16 & 0 & 2.53 & 9.16 & 0 & 2.53 & 9.16 & 0 & 2.53 & 9.16 & 0 & 2.53 \\
\hline $\mathrm{I}$ & 15.27 & 0 & 1.90 & 15.27 & 0 & 1.90 & 15.27 & 0 & 1.90 & 15.27 & 15.27 & 0 & 15.27 & 0 & 1.90 \\
\hline $\mathrm{J}$ & 7.56 & 0 & 1.18 & 7.56 & 0 & 1.18 & 7.56 & 0 & 1.18 & 7.56 & 0 & 1.18 & 7.56 & 0 & 1.18 \\
\hline $\mathrm{K}$ & 9.81 & 0 & 2.47 & 9.81 & 0 & 2.47 & 9.81 & 0 & 2.47 & 9.81 & 0 & 2.47 & 9.81 & 0 & 2.47 \\
\hline $\mathrm{L}$ & 10.63 & 0 & 1.82 & 10.63 & 0 & 1.82 & 10.63 & 10.63 & 0 & 10.63 & 0 & 1.82 & 10.63 & 0 & 1.82 \\
\hline $\mathrm{M}$ & 11.00 & 0 & 2.24 & 11.00 & 0 & 2.24 & 11.00 & 0 & 2.24 & 11.00 & 0 & 2.24 & 11.00 & 0 & 2.24 \\
\hline $\mathrm{N}$ & 12.13 & 0 & 2.26 & 12.13 & 0 & 2.26 & 12.13 & 0 & 2.26 & 12.13 & 0 & 2.26 & 12.13 & 12.13 & 0 \\
\hline $\mathrm{O}$ & 12.14 & 0 & 1.45 & 12.14 & 0 & 1.45 & 12.14 & 0 & 1.45 & 12.14 & 0 & 1.45 & 12.14 & 0 & 1.45 \\
\hline $\mathrm{P}$ & 11.99 & 0 & 1.26 & 11.99 & 0 & 1.26 & 11.99 & 0 & 1.26 & 11.99 & 0 & 1.26 & 11.99 & 0 & 1.26 \\
\hline $\mathrm{Q}$ & 10.42 & 0 & 2.75 & 10.42 & 0 & 2.75 & 10.42 & 0 & 2.75 & 10.42 & 0 & 2.75 & 10.42 & 0 & 2.75 \\
\hline $\mathrm{R}$ & 13.43 & 0 & 1.48 & 13.43 & 0 & 1.48 & 13.43 & 0 & 1.48 & 13.43 & 0 & 1.48 & 13.43 & 0 & 1.48 \\
\hline$S$ & 7.94 & 0 & 1.33 & 7.94 & 0 & 1.33 & 7.94 & 0 & 1.33 & 7.94 & 0 & 1.33 & 7.94 & 0 & 1.33 \\
\hline $\mathrm{T}$ & 7.56 & 0 & 2.18 & 7.56 & 0 & 2.18 & 7.56 & 0 & 2.18 & 7.56 & 0 & 2.18 & 7.56 & 0 & 2.18 \\
\hline \multirow[t]{2}{*}{ Sum } & 218.36 & 12.07 & 38.02 & 218.36 & 11.47 & 37.42 & 218.36 & 10.63 & 38.27 & 218.36 & 15.27 & 38.19 & 218.36 & 12.13 & 37.83 \\
\hline & $E_{\mathrm{d}}$ & \multicolumn{2}{|c|}{$E_{\mathrm{d}}+E_{\mathrm{s}}$} & $E_{\mathrm{d}}$ & \multicolumn{2}{|c|}{$E_{\mathrm{d}}+E_{\mathrm{s}}$} & $E_{\mathrm{d}}$ & \multicolumn{2}{|c|}{$E_{\mathrm{d}}+E_{\mathrm{s}}$} & $E_{\mathrm{d}}$ & $E_{\mathrm{d}}+$ & $+E_{\mathrm{S}}$ & $E_{\mathrm{d}}$ & \multicolumn{2}{|c|}{$E_{\mathrm{d}}+E_{\mathrm{s}}$} \\
\hline Total & 218.36 & \multicolumn{2}{|c|}{50.09} & 218.36 & \multicolumn{2}{|c|}{48.89} & 218.36 & \multicolumn{2}{|c|}{48.9} & 218.36 & 53. & 46 & 218.36 & \multicolumn{2}{|c|}{49.96} \\
\hline Ip. Ratio & \multicolumn{3}{|c|}{$77.06 \%$} & & 77.6 & $1 \%$ & & 77.6 & $51 \%$ & & 75.5 & $2 \%$ & & 77.1 & $2 \%$ \\
\hline
\end{tabular}




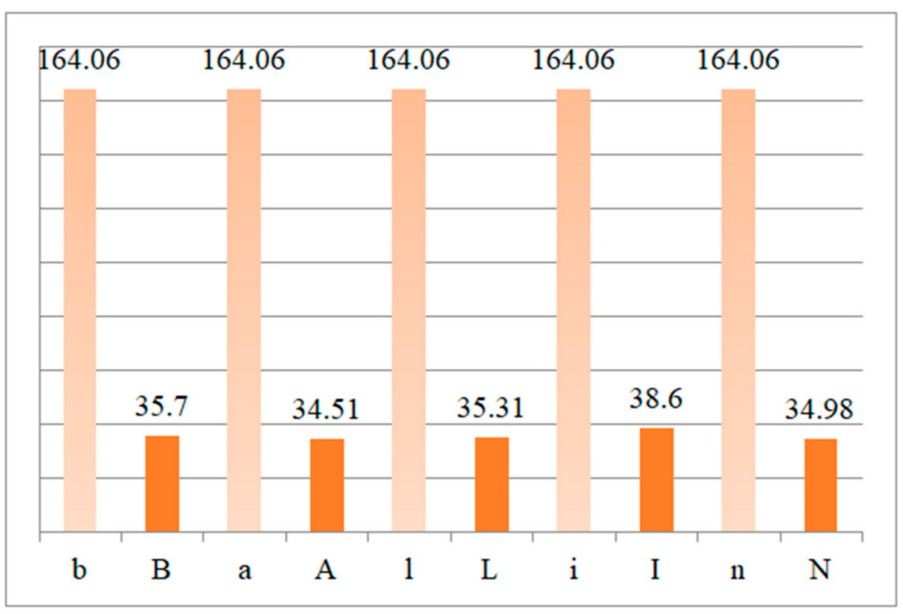

Figure 7. Comparison of the energy consumption of three cameras, one set using the current method, and one set using the EEC approach.

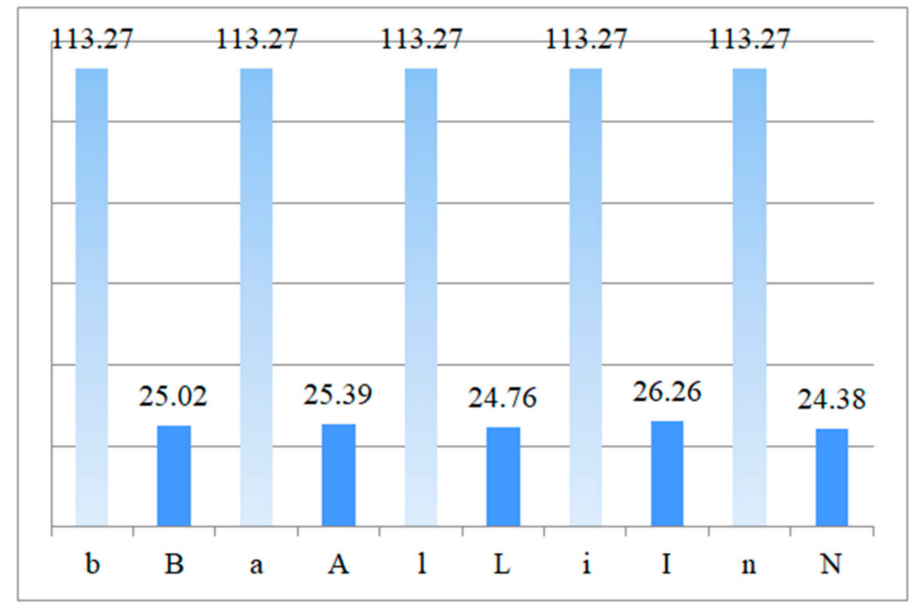

Figure 8. Comparison of the energy consumption of two cameras, one set using the current method, and one set using the EEC approach.

\section{Conclusions and Future Work}

Object tracking systems used to collect traffic data have evolved to meet the demands of the present-day smart city, which relies on data monitoring for traffic, security, and crime applications. Widespread construction of object tracking systems results in high energy costs. This work focuses on object tracking networks to propose EEC that achieve energy savings. First, we analyzed the architecture of object tracking systems. After that, we presented an equivalent model of an object tracking system. Next, we defined a cluster structure that consists of a number of object tracking devices for improving the system's viewing coverage. For the accurate assessment of energy costs, we accounted for dynamic and static energy consumption during active and standby modes, respectively. Then, we derived several formulas that facilitate a straightforward evaluation of energy costs. Finally, simulation results were reported, which show that the proposed approach effectively saved energy compared to the given simulation benchmarks.

Object tracking systems are continually studied in academic and commercial fields, with the goal of providing fast services and more accurate results. Moreover, there is a trend toward embedding new technology, such as fog computing or 5G mobile function, into object tracking systems to enable fog services. These services consume more energy in comparison with current technology. As a result, efficient energy consumption for object tracking systems will continue to be a significant issue in the future. 
Funding: This research was supported by the Ministry of Science and Technology, Taiwan, grant number MOST 106-2221-E-143-002.

Conflicts of Interest: The author declares no conflict of interest.

\section{References}

1. Auto-Follow Camera, Movensee Company. Available online: https://shop.movensee.com/en/?gclid= EAIaIQobChMIh6jqr6aY2AIVVSUrCh2OrQizEAAYAyAAEgL0J_D_BwE (accessed on 21 May 2018).

2. Smart Multiple Target Tracking Camera, Rayoptic Company. Available online: http://www.rayphotonics.com/Multi-target-intelligent-tracking--system?gclid= EAIaIQobChMIr7bShsCY2AIV1zUrCh2EvQ1cEAAYAyAAEgIuAPD_BwE (accessed on 21 May 2018).

3. Wang, M.-L.; Huang, C.-C.; Lin, H.-Y. An Intelligent Surveillance System Based on an Omnidirectional Vision Sensor. In Proceedings of the 2006 IEEE Conference on Cybernetics and Intelligent Systems, Bangkok, Thailand, 7-9 June 2006.

4. Chang, K.-C.; Liu, P.-K.; Yu, C.-S. Design of Real-Time Video Streaming and Object Tracking System for Home Care Services. In Proceedings of the 2016 IEEE International Conference on Consumer Electronics-Taiwan, Nantou, Taiwan, 27-29 May 2016.

5. Liu, M.; Sui, H.; Wang, J.; Wu, C. Study of Intelligent Video Monitoring Technology. In Proceedings of the 2010 Chinese Control and Decision Conference, Xuzhou, China, 26-28 May 2010.

6. Boudjit, K.; Larbes, C. Detection and Implementation Autonomous Target Tracking with a Quadrotor AR.Drone. In Proceedings of the 2015 12th International Conference on Informatics in Control, Automation and Robotics (ICINCO), Colmar, France, 21-23 July 2015.

7. Yu, X.; Tu, X.; Ang, E.L. Trajectory-Based Ball Detection and Tracking in Broadcast Soccer Video with the Aid of Camera Motion Recovery. In Proceedings of the 2007 IEEE International Conference on Multimedia and Expo, Beijing, China, 2-5 July 2007.

8. Jiang, L.; Carlo, S. Tracking Multiple Moving Objects in Images Using Markov Chain Monte Carlo. Available online: https: / /arxiv.org/abs/1603.05522,2016 (accessed on 21 May 2018).

9. Fang, C.Y.; Lo, C.S.; Ho, S.H.; Chuang, S.H.; Chen, S.W. A Vision-Based Infant Monitoring System Using PT IP Camera. In Proceedings of the 2016 International Symposium on Computer, Consumer and Control, Xi'an, China, 4-6 July 2016.

10. Chellappa, R.; Qian, G.; Zheng, Q. Vehicle Detection and Tracking Using Acoustic and Video Sensors. In Proceedings of the 2004 IEEE International Conference on Acoustics, Speech, and Signal, Montreal, QC, Canada, 17-21 May 2004.

11. Pan, P.; Schonfeld, D. Visual Tracking Using High-Order Particle Filtering. IEEE Signal Process. Lett. 2011, 18, 51-54. [CrossRef]

12. Kurazume, R.; Yamada, H.; Murakami, K.; Iwashita, Y.; Hasegawa, T. Target Tracking Using SIR and MCMC Particle Filters by Multiple Cameras and Laser Range Finders. In Proceedings of the 2008 IEEE/RSJ International Conference on Intelligent Robots and Systems, Nice, France, 22-26 September 2008.

13. Ge, Z.; Chang, F.; Liu, H. Multi-target Tracking based on Kalman Filtering and Optical Flow Histogram. In Proceedings of the 2017 Chinese Automation Congress (CAC), Jinan, China, 20-22 October 2017.

14. Li, J.; Ye, D.H.; Chung, T.; Kolsch, M.; Wachs, J.; Bouman, C. Multi-target Detection and Tracking from a Single Camera in Unmanned Aerial Vehicles (UAVs). In Proceedings of the 2016 IEEE/RSJ International Conference on Intelligent Robots and Systems (IROS), Daejeon, Korea, 9-14 October 2016.

15. Surana, A.; Nakhmani, A.; Tannenbaum, A. Anomaly Detection in Videos: A Dynamical Systems Approach. In Proceedings of the 52nd IEEE Conference on Decision and Control, Firenze, Italy, 10-13 December 2013.

16. Duffner, S.; Garcia, C. Using Discriminative Motion Context for Online Visual Object Tracking. IEEE Trans. Circuits Syst. Video Technol. 2016, 26, 2215-2225. [CrossRef]

17. Zhang, W.; Tian, X. New Method of Object Tracking under Complex Circumstance. In Proceedings of the 2010 International Conference on E-Business and E-Government, Guangzhou, China, 7-9 May 2010.

18. Li, G.; Qu, W.; Huang, Q. A Multiple Targets Appearance Tracker Based on Object Interaction Models. IEEE Trans. Circuits Syst. Video Technol. 2012, 22, 450-464. [CrossRef]

19. Kang, B.; Zhu, W.P.; Liang, D. Robust m=Multi-feature Visual Tracking via Multi-task Kernel-based Sparse Learning. IET Image Process. 2017, 11, 1172-1178. [CrossRef] 
20. Lee, J.; Seo, Y.W.; Wettergreen, D. Kernel-based Tracking for Improving Sign Detection Performance. In Proceedings of the 2013 IEEE/RSJ International Conference on Intelligent Robots and Systems, Tokyo, Japan, 3-7 November 2013.

21. Babu, R.V.; Perez, P.; Bouthemy, P. Robust Tracking with Motion Estimation and Kernel-based Color Modelling. In Proceedings of the IEEE International Conference on Image Processing, Genova, Italy, 11-14 September 2005.

22. Pooranian, Z.; Abawajy, J.H.; Vinod, P.; Conti, M. Scheduling Distributed Energy Resource Operation and Daily Power Consumption for a Smart Building to Optimize Economic and Environmental Parameters. Energies 2018, 11, 1348. [CrossRef]

23. Baccarelli, E.; Naranjo, P.G.V.; Scarpiniti, M.; Shojafar, M.; Abawajy, J.H. Fog of Everything: Energy-Efficient Networked Computing Architectures, Research Challenges, and a Case Study. IEEE Access 2017, 5, 9882-9910. [CrossRef]

24. Naranjo, P.G.V.; Pooranian, Z.; Shojafar, M.; Conti, M.; Buyya, R. FOCAN: A Fog-supported Smart City Network Architecture for Management of Applications in the Internet of Everything Environments. J. Parallel Distrib. Comput. 2018, 7. [CrossRef]

25. Fan, Y.-H. Energy-Aware Path Strategy for Embedded Network. Int. J. Distrib. Sens. Netw. 2017, $13,1-11$. [CrossRef]

26. Fan, Y.-H.; Wu, J.-O.; Wang, S.-F. Energy Efficiency of Task Allocation for Embedded JPEG Systems. Sci. World J. 2014, 2014, 718348. [CrossRef] [PubMed]

27. Fan, Y.-H.; Wu, J.-O.; Wang, S.-F. Low Power Dissipation Model Analysis for Embedded Systems. J. Res. Notes Inf. Sci. 2013, 13, 184-188.

28. AHD 4MP Surveillance Camera, Kingnet Technology Company. Available online: http:/ /www.king-net. com/content.php?cn=item\&tid=1349\&start=0 (accessed on 21 May 2018).

(C) 2018 by the author. Licensee MDPI, Basel, Switzerland. This article is an open access article distributed under the terms and conditions of the Creative Commons Attribution (CC BY) license (http:/ / creativecommons.org/licenses/by/4.0/). 